\title{
Full-waveform inversion with an exponential filter in wavenumber domain
} \author{
Kai Chang ${ }^{\dagger}$, Chao Song ${ }^{\ddagger}$, Tariq Alkhalifa $h^{\ddagger}$ and Haijiang Zhang ${ }^{\dagger}$, University of Science and Technology of China ${ }^{\dagger}$,
} King Abdullah University of Science and Technology $y^{\ddagger}$

\section{SUMMARY}

The gradient of full-waveform inversion (FWI) can be decomposed into a tomographic part, which is smooth (lowwavenumbers) and an image part, which is more sharp (highwavenumbers). Most of the FWI procedures need to update the smooth part first and gradually include the details to mitigate the cycle-skipping problem. The scattering angle filter for the gradient is one way to isolate the low-wavenumber part of the gradient at the early stage. However, it could be costly because of the extensions necessary for precise scattering angle control. Due to the relationship between the wavenumber and the scattering angle, the large scattering angles correspond to the low wavenumber component, which is usually what we want to update in our initial inversion steps. Thus, we use an exponential filter in the wavenumber domain and update the model in the wavenumber domain from low to high. The numerical result indicates that the inversion benefits from the wavenumber domain filter.

\section{INTRODUCTION}

Full-waveform inversion has attracted a lot of attention because it can admit high-resolution velocity models. In the process of FWI, obtaining a macro-smooth model at the beginning is critical to the inversion. If such a model is not be obtained, FWI often fails. The traditional multi-scale FWI mitigates the cycle-skipping issue by inverting the model from low to high frequencies gradually in the data domain (Pratt, 1999). However, the required low-frequency information necessary in reality to generate a sufficiently smooth model update is often missing. Thus, many methods have been proposed: to address this problem from the data side, such as low-frequency extrapolation (Li and Demanet, 2016; Ovcharenko et al., 2019) or early arrivals full waveform inversion (Bleibinhaus et al., 2009). From the perspective of modifying the objective function, wavefield reconstruction inversion (WRI) and efficient wavefield inversion (EWI) have been proposed, add a regularization parameter and expand the solution space (Van Leeuwen and Herrmann, 2013; Alkhalifah and Song, 2019; Song and Alkhalifah, 2020); reflection waveform inversion (RWI), which based on the Born approximation, using the migration and the demigration procedure to build up a good initial velocity model (Xu et al., 2012; Wang et al., 2017; Guo and Alkhalifah, 2017). In addition, multi-scattering waveform inversion (MWI) was applied successfully to many cases (Alkhalifah and Wu, 2016; Alkhalifah, 2016; Song et al., 2019).

According to the nature of the seismic wave itself, the scattering angle between the source and the receiver wavefields determines the wavenumber component of the gradient. The scattering angle along the ray path between the source and the receiver is close to 180 degrees, referred to as the Direct Cur- rent (DC) component of the wavenumber. In order to obtain low wavenumber components at the early iterations, different filters are designed. Energy norm imaging condition, which is equivalent to a Laplacian filter was applied to the FWI (Rocha et al., 2016; Sun and Alkhalifah, 2017). However, the filter cannot obtain a sufficiently low wavenumber gradient for the second order decay corresponding to the scattering angle cosine value. Xie (2015) applied an angle-domain wavenumber filter for multi-scale full-waveform inversion by the decomposed source and receiver wavefield into local plane waves. It can be costly to decompose the wavefield. Different from dealing with the wavefield, Alkhalifah (2014) suggested filtering the gradient by using a space extension which can be free of a velocity dependency and thus becomes a stationary filter. However, it still can be expensive as it rely on the extensions, especially for 3D cases.

To obtain an efficient scattering angle filter, interpolation can be used to transform the space lag to a velocity lag (Alkhalifah, 2015; Kazei et al., 2016). Wu and Alkhalifah (2017) proposed to use an enhanced scattering-angle enrichment to achieve the extraction of low wavenumber components efficiently. However, this method will have singular values when the wavenumber equals zero.

To mitigate this issue, we propose to use an exponential filter, which naturally decays as the wavenumber increases. The structure of this abstract is as follows. First, we derive the scattering-angle enrichment method and then replace the filter factor. We use the proposed filter to the gradient and then invert the Marmousi model with a single $5 \mathrm{~Hz}$ frequency from low to high wavenumbers gradually. At last, We show the effectiveness of this method for multiple frequencies.

\section{THEORY}

We will first review the scattering-angle enrichment method, and then replace the wavenumber filter factor as well as normalize it. The gradient of FWI $I(x)$ corresponding to the forward wavefield $u_{s}(x, t)$ and its adjoint $u_{r}(x, t)$ can be written $I(x, t)=\int_{t} u_{s}(x, t) u_{r}(x, t) d t$, The time extend image $I(x, \tau)$ with a time extention $\tau$ can be defined as:

$$
\begin{aligned}
I(x, \tau) & =\int_{t} u_{s}(x, t-\tau) u_{r}(x, t+\tau) \mathrm{d} t \\
& =\int_{\omega_{t}} U_{s}\left(x, \omega_{t}\right) \bar{U}_{r}\left(x, \omega_{t}\right) e^{2 i \omega_{t} \tau} \mathrm{d} \omega_{t},
\end{aligned}
$$

where the $U_{s}$ and $\bar{U}_{r}$ are the forward wavefield and the adjoint wavefield in frequency domain, respectively. And the ${ }^{-}$means the complex conjugate operator. $\omega_{t}$ is the temporal frequency of the seismic data. 


\section{FWI with an exponential filter in wavenumber domain}

And we also define the gradient spectrum $I\left(k_{x}, \omega_{\tau}\right)=F F T(I(x, \tau))$, where the FFT operator indicates the fast Fourier transform in all dimensions, $k_{x}$ is the wavenumber, $\omega_{\tau}$ is the frequency index of $\tau$. For the inverse Fourier transform, the precise gradient $I(x)$ is the integral over the $\omega_{\tau}$ and the inverse Fourier transform of the $k_{x}$ to the space domain, which can be written as:

$$
I(x)=I(x, 0)=\frac{1}{\sqrt{2 \pi}^{D+1}} \int_{\omega_{\tau}} \int_{k_{x}} I\left(k_{x}, \omega_{\tau}\right) e^{i k_{x} \cdot x} \mathrm{~d} k_{x} \mathrm{~d} \omega_{\tau},
$$

where $\mathrm{D}$ is the dimension of the space domain. Our objective is to add a filter for isolating the low wavenumber part (i.e. the large scattering angle part) of the gradient. The filtered gradient can be written:

$$
I_{f}(x)=\frac{1}{\sqrt{2 \pi}^{D+1}} \int_{\omega_{\tau}} \int_{k_{x}} F(\theta) I\left(k_{x}, \omega_{\tau}\right) e^{i k_{x} \cdot x} \mathrm{~d} k_{x} \mathrm{~d} \omega_{\tau} .
$$

$F(\theta)$ is the filter that relies on the half of scattering angle $\theta$. For the dispersion equation, the relationship between the $\theta$ and local wavenumber is

$$
\cos (\theta)=\frac{1}{2} \frac{\left|k_{x}\right| v}{\left|\omega_{\tau}\right|}
$$

The main problem in equation 3 is that the calculation of $I\left(k_{x}, \omega_{\tau}\right)$ with the time extension $\tau$ can be costly. Wu and Alkhalifah(2017) came up with an idea that uses $\cos ^{n}(\theta)$ to enrich the filter, where $n$ is a particular number. When $n<0$, we obtain the low wavenumber components and when $n>0$, we can get the high wavenumber part of the gradient. Specifically, when $n=0$, the filter turns to be trivial. Then the filtered gradient can be written:

$$
I_{f}^{n}(x)=\frac{v^{n}}{\sqrt{2 \pi}^{D+1}} \int_{\omega_{\tau}} \int_{k_{x}} \frac{\left|k_{x}\right|^{n}}{\left|\omega_{\tau}\right|^{n}} I\left(k_{x}, \omega_{\tau}\right) e^{i k_{x} \cdot x} \mathrm{~d} k_{x} \mathrm{~d} \omega_{\tau} .
$$

To avoid the large amount of calculation, according to equation 2 and equation 1 , we combine the $\left|\omega_{\tau}\right|^{n}$ and $I\left(k_{x}, \omega_{\tau}\right)$, then integrate over $\omega_{\tau}$ and $\tau$. We finally get the filtered gradient without the time shift $\tau$ :

$$
I_{f}^{n}(x)=\frac{v^{n}}{\sqrt{2 \pi}{ }^{D} 2^{n}} \int_{k_{x}} \int_{\omega_{t}} \frac{\left|k_{x}\right|^{n}}{\omega_{t}^{n}} \hat{\tilde{I}}\left(k_{x}, \omega_{t}\right) e^{i k_{x} \cdot x} \mathrm{~d} \omega_{t} \mathrm{~d} k_{x} .
$$

$\hat{\bar{I}}\left(k_{x}, \omega_{t}\right)=F F T(I(x, t))$. In equation (6), we can get a singularity when $\left|k_{x}\right|=0$ at denominator, which is the DC component. To mitigate the issue, alternatively, neglecting the weighting coefficient of the Fourier transform, we apply an exponential filter to the gradient as follows,

$$
I_{f}^{\alpha}(x)=\int_{k_{x}} \int_{\omega_{t}} e^{-\alpha\left|k_{x}\right|} \hat{\tilde{I}}\left(k_{x}, \omega_{t}\right) e^{i k_{x} \cdot x} \mathrm{~d} \omega_{t} \mathrm{~d} k_{x}
$$

The exponential filter has an advantage that it is naturally normalized and always less than one. Figure 1 demonstrates that the different features of the two filters. The DC component in Figure 1a is muted to avoid the singularity, and the exponential filter in Figure 1b mitigates this issue.

The workflow of suggested filter is given in Algorithm 1.
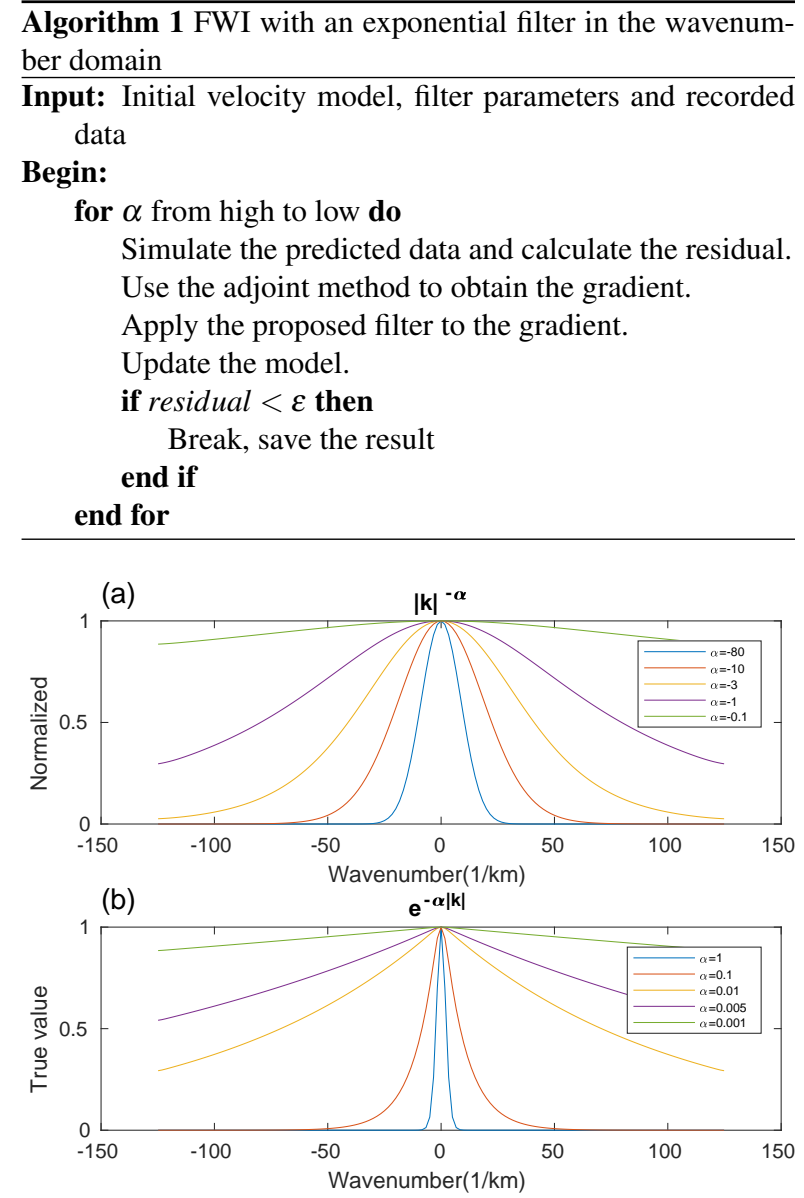

Figure 1: (a) The normalized filter of the $\cos ^{n}(\theta)$ in wavenumber domain. (b) The exponential filter in the wavenumber domain.

\section{EXAMPLE}

We show the effectiveness of this exponential filtering method in FWI through numerical results.

\section{Kernel Analysis}

First, we use a homogeneous model to illustrate the method. The true model is a homogeneous model and its velocity equals to $3.5 \mathrm{~km} / \mathrm{s}$. The initial model is a gradient model from $2 \mathrm{~km} / \mathrm{s}$ to $3.6 \mathrm{~km} / \mathrm{s}$. The size of the model is $351 \times 141$ with grid interval of $25 \mathrm{~m}$ in both directions. We use one source and one receiver on the surface. The source and receiver are located at the $\mathrm{x}=1.75 \mathrm{~km}$ and $\mathrm{x}=7 \mathrm{~km}$, respectively. We generate the data in the frequency domain with a single frequency of $10 \mathrm{~Hz}$. Then we use the data residual to get the gradient by the adjoint state method (Pratt, 1999). Figure 2a shows the gradient of the FWI, and we can see both the low wavenumber and high wavenumber components are mixed in space. The proposed filtering method is utilized to gradually filter out the high wavenumber components. Figure $2 b-d$ show the effectiveness of different filter factors on the gradient. The larger $\alpha$ admits lower wavenumbers in the gradient. 
FWI with an exponential filter in wavenumber domain

\section{The Marmousi Model}

In order to prove the effectiveness of our method on a complex velocity model, we apply the method to the Marmousi model (Figure 3a). The initial velocity model is linearly increasing with depth, $v(z)=1.5+0.8 \times z(\mathrm{~km} / \mathrm{s})$ (Figure $3 \mathrm{~b}$ ). The size of the Marmousi model is the asme as the previous model we used in the sensitivity kernel. we have 71 shots on the surface with the source interval is $125 \mathrm{~m}$ and the receiver is fully covered at the surface with a $25 \mathrm{~m}$ interval.

Due to the high efficiency of frequency domain interpretations, we first use a mono frequency of $5 \mathrm{~Hz}$ to demonstrate the effectiveness of our method. As shown in Figures $4 a-c$, we use the exponential filtering parameter to gradually update the velocity model from low wavenumber to high wavenumber. Compared with the mono frequency traditional FWI result (Figure 4d), which has strong artefacts, falling into a local minimum, we can conclude that the filter can mitigate the cycle skipping issue even at $5 \mathrm{~Hz}$ data. We used the L-BFGS method for optimizing the inverse problem. Every wavenumber filter stage has a maximum of 100 iterations. Actually, we achieve to get convergence at the early stage of the iterations for the smoother gradients. We suggest the filter parameter $\alpha$ starts from 1 to 0 for inverting the Marmousi model.

We also used multi-frequencies from $5 \mathrm{~Hz}$ to $12 \mathrm{~Hz}$ with a $1 \mathrm{~Hz}$ interval and $7 \mathrm{~Hz}$ dominant frequency Using the same inversion setup parameters, we can get the clean velocity structure even at the deep part of the model (Figure 5a). In contrast, the traditional FWI falls into a local minimum reflected by the poor result (Figure $5 b$ ).

\section{CONCLUSION}

Obtaining the low wavenumber part of the gradient is an effective way to mitigate the cycle skipping problem. We proposed an exponential filter in the wavenumber domain, which can isolate the low wavenumber in the FWI procedure. We applied the method to both a homogeneous model and the Marmousi model, gradually filtering the gradient wavenumber from low to high. The results indicate that using the filter can result in a good initial background velocity and subsequently an accurate velocity model.

\section{ACKNOWLEDGMENTS}

We would like to thank KAUST for supporting the study and Seismic Wave Analysis Group (SWAG) for fruitful discussions. The author Kai Chang also thanks to the China Scholar Council (CSC) for supporting his study in KAUST.
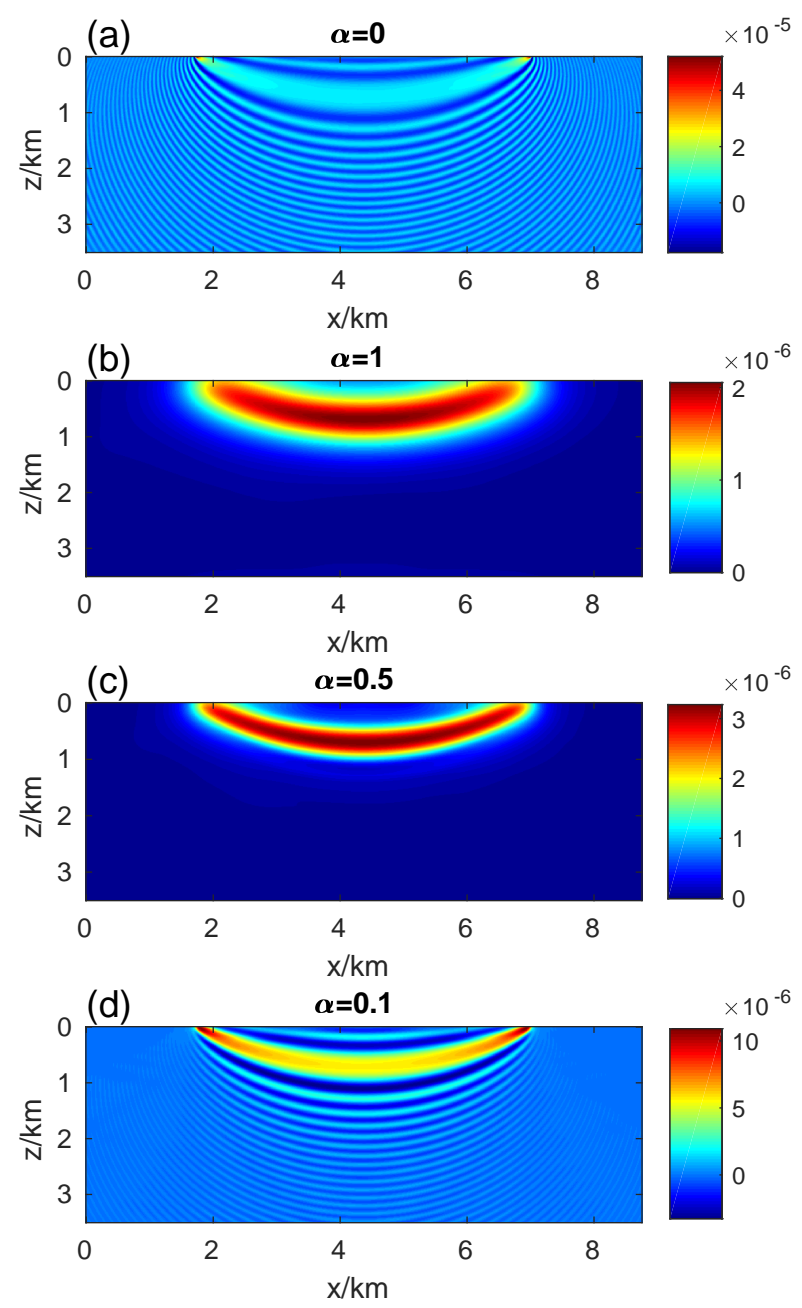

Figure 2: The original kernel (a) and the filtered gradient by the exponential filter with $\alpha=1(\mathrm{~b}), \alpha=0.5(\mathrm{c}), \alpha=0.1(\mathrm{~d})$.
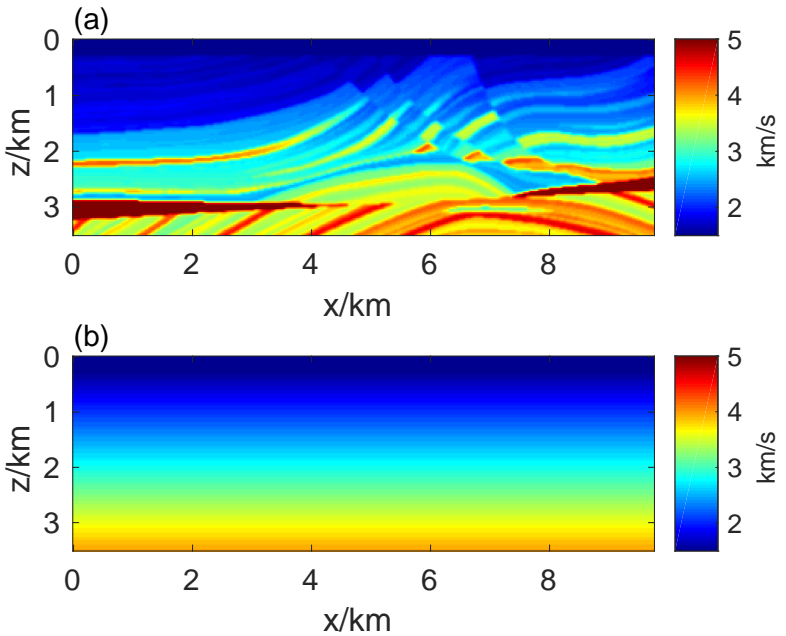

Figure 3: (a) The true Marmousi model, (b) the initial model we used in this study. 

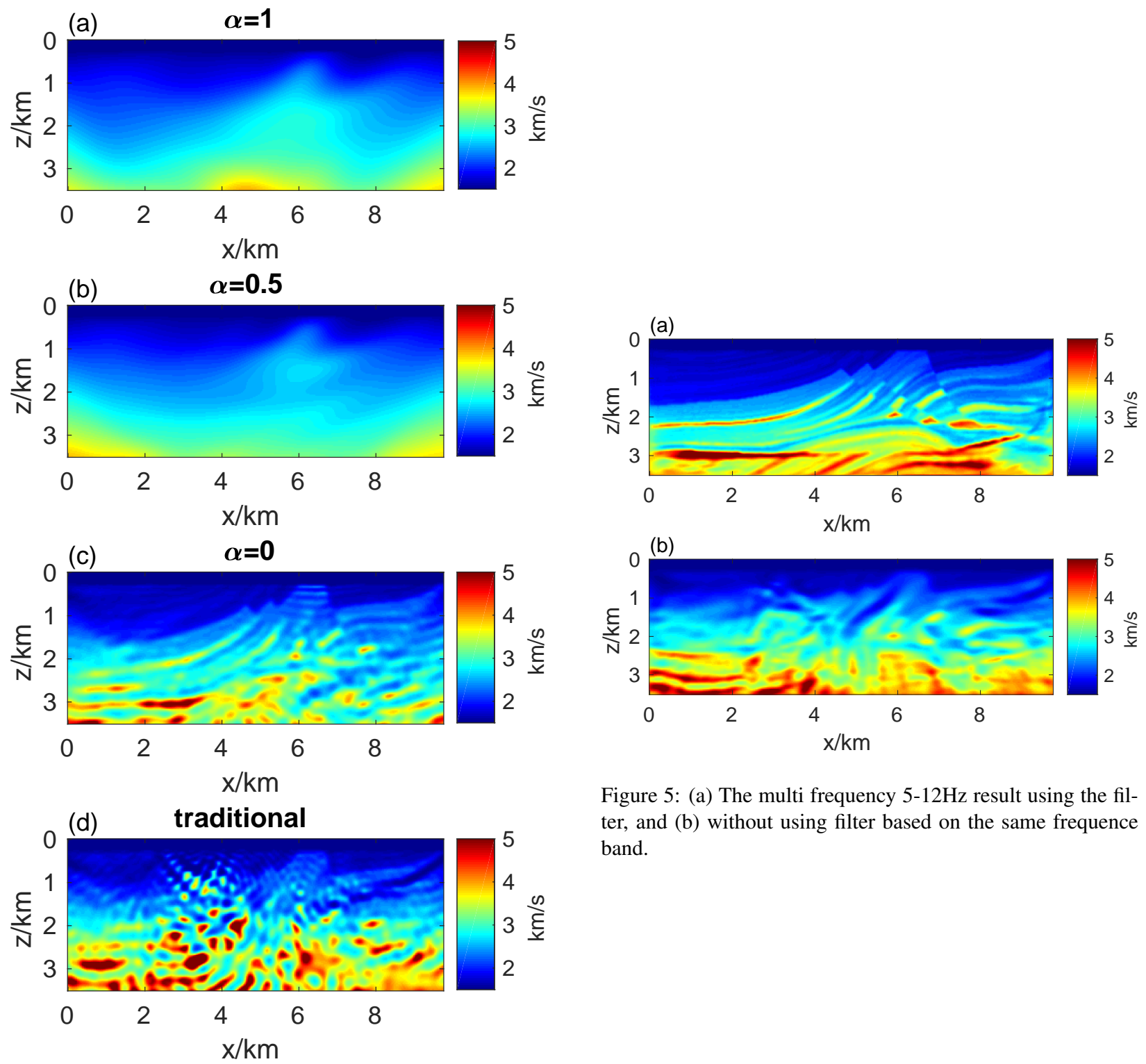

Figure 5: (a) The multi frequency $5-12 \mathrm{~Hz}$ result using the filter, and (b) without using filter based on the same frequence band.

Figure 4: The inversion result using the wavenumber filter factor $\alpha=1(\mathrm{a}), \alpha=0.5$ (b) and $\alpha=0$ (c), sequentially. (d) is the traditional full waveform inversion result. 


\section{FWI with an exponential filter in wavenumber domain}

\section{REFERENCES}

Alkhalifah, T., 2014, Scattering-angle based filtering of the waveform inversion gradients: Geophysical Journal International, 200, 363-373.

— 2015 , Efficient scattering angle filtering for full waveform inversion, in SEG Technical Program Expanded Abstracts 2015: Society of Exploration Geophysicists, 1138-1142.

— , 2016, Full-model wavenumber inversion: An emphasis on the appropriate wavenumber continuation: Geophysics, 81, R89-R98.

Alkhalifah, T., and C. Song, 2019, An efficient wavefield inversion: Using a modified source function in the wave equation: Geophysics, 84, R909-R922.

Alkhalifah, T., and Z. Wu, 2016, Multiscattering inversion for low-model wavenumbers: Geophysics, 81, R417-R428.

Bleibinhaus, F., R. W. Lester, and J. A. Hole, 2009, Applying waveform inversion to wide-angle seismic surveys: Tectonophysics, 472, 238-248.

Guo, Q., and T. Alkhalifah, 2017, Elastic reflection-based waveform inversion with a nonlinear approach: Geophysics, 82, R309R321.

Kazei, V., E. Tessmer, and T. Alkhalifah, 2016, Scattering angle-based filtering via extension in velocity, in SEG Technical Program Expanded Abstracts 2016: Society of Exploration Geophysicists, 1157-1162.

Li, Y. E., and L. Demanet, 2016, Full-waveform inversion with extrapolated low-frequency data: Geophysics, 81, R339-R348.

Ovcharenko, O., V. Kazei, M. Kalita, D. Peter, and T. Alkhalifah, 2019, Deep learning for low-frequency extrapolation from multioffset seismic data: Geophysics, 84, R989-R1001.

Pratt, R. G., 1999, Seismic waveform inversion in the frequency domain, part 1: Theory and verification in a physical scale model: Geophysics, 64, 888-901.

Rocha, D., N. Tanushev, and P. Sava, 2016, Acoustic wavefield imaging using the energy norm: Geophysics, 81, S151-S163.

Song, C., and T. A. Alkhalifah, 2020, Efficient wavefield inversion with outer iterations and total variation constraint: IEEE Transactions on Geoscience and Remote Sensing.

Song, C., Z. Wu, and T. Alkhalifah, 2019, Passive seismic event estimation using multiscattering waveform inversion: Geophysics, 84, KS59-KS69.

Sun, B., and T. Alkhalifah, 2017, Wavefield separation by energy norm born scattering, in SEG Technical Program Expanded Abstracts 2017: Society of Exploration Geophysicists, 1481-1485.

Van Leeuwen, T., and F. J. Herrmann, 2013, Mitigating local minima in full-waveform inversion by expanding the search space: Geophysical Journal International, 195, 661-667.

Wang, G., S. Wang, Q. Du, and S. Yuan, 2017, Traveltime-based reflection full-waveform inversion for elastic medium: Journal of Applied Geophysics, 141, 68-76.

Wu, Z., and T. Alkhalifah, 2017, Efficient scattering-angle enrichment for a nonlinear inversion of the background and perturbations components of a velocity model: Geophysical Journal International, 210, 1981-1992.

Xie, X.-B., 2015, An angle-domain wavenumber filter for multi-scale full-waveform inversion, in SEG technical program expanded abstracts 2015: Society of Exploration Geophysicists, 1132-1137.

Xu, S., D. Wang, F. Chen, Y. Zhang, and G. Lambare, 2012, Full waveform inversion for reflected seismic data: 74th EAGE Conference and Exhibition incorporating EUROPEC 2012, European Association of Geoscientists \& Engineers, cp-293. 